\title{
Another Approach to Save Energy in OFDM Systems
}

\author{
Xiaoying Shao, Cornelis H. Slump \\ University of Twente, Enschede, the Netherlands \\ E-mail: \{x.shao, c.h.slump\}@ewi.utwente.nl
}

\begin{abstract}
In this paper, we propose an energy-efficient error correction scheme to lower the power consumption of the ADCs in the OFDM system. The proposed opportunistic error correction scheme is based on resolution adaptive ADCs and fountain codes. The key idea is to reduce the dynamic range of the channel by discarding part of the channel in deep fading. Correspondingly, the power consumption in ADCs can be decreased. In our approach, each sub-carrier transports a fountain-encoded packet. The receiver only decodes fountainencoded packets with high SNR. Others are discarded. To compensate for the discarded packets, a high order modulation is used. The new error correction layer does not require perfect channel knowledge, so it can be applied in a real system. With our approach and 16-QAM, the energy consumption in ADCs is reduced by around $73 \%$ with non-perfect channel estimation comparing to the traditional IEEE 802.11a system under the same channel conditions and throughput.
\end{abstract}

\section{INTRODUCTION}

Orthogonal Frequency Division Multiplexing (OFDM) is a common technology to ease the equalizer in wide-band communication [1]. The disadvantage of OFDM is its high Peak-to-Average Power Ratio (PAPR). When signal peaks in the OFDM signal are clipped, all sub-carriers are affected after the OFDM demodulation. Due to the frequency selective characteristics of wireless channels, some sub-carriers suffer deep fading and can not endure any distortion. That urges the usage of high-resolution Analog-to-Digital Converters (ADCs) in the OFDM system.

In wireless $\mathrm{LAN}$ receivers, the $\mathrm{ADC}$ can consume up to $50 \%$ of the total baseband power budget [2]. Lower power consumption in battery-powered wireless receivers is a highly desirable feature. Consumers expect their devices to operate for several hours on a single battery charge. The power efficiency of ADCs does not increase over time in the same way as baseband signal processing. Given the same specification, the power consumed in ADCs halves every 2.7 years [3] but the power dissipation in the baseband signal processing decreases a factor of 10 every 5 years [4]. In the case of RF signal processing, its power efficiency is limited by the semiconductor technology. Therefore, ADCs are the main bottleneck for an energy-efficient wireless receiver.

In this paper, we propose a novel cross layer scheme to reduce the power consumption of ADCs by combining a resolution adaptive ADC architecture with Opportunistic Error
Correction (OEC). Current OFDM systems utilize fixed highresolution ADCs, which are designed for the worst-case channel condition. However, worst-case scenarios do not happen often. Resolution adaptive ADCs allow us to adapt the ADC for each channel condition instead of being fixed for the worstcase condition. Correspondingly, the power consumption in ADCs can be reduced.

A further power reduction can be achieved by discarding some parts of the channel in deep fading. Current OFDM systems utilize the joint coding scheme which encodes source data over all the sub-carriers. The joint coding scheme works better than the separate coding scheme, as it employs the fact that sub-carriers with high energy can compensate for those in deep fading [1]. However, it treats each sub-carrier equally important. Hence, the minimum resolution of ADC required by the joint coding scheme is proportional to the dynamic range of the channel. In many frequency selective channels, only a small part of the channel causes the high dynamic range. Let us take Fig. 1 as an example, the dynamic range of the whole channel is around $16 \mathrm{~dB}$. As we can see, the deep fading part occupies a large part of the dynamic range, but deep fading only happens in the frequency band of $-1 \sim 0.5 \mathrm{MHz}$. By discarding this $1.5 \mathrm{MHz}$ sub-band, the dynamic range of the channel is reduced by $50 \%$ (i.e. around $8 \mathrm{~dB}$ ). Obviously, the joint coding scheme does not allow us to do it. But with fountain codes, we can achieve it.

In this paper, we propose opportunistic error correction based on fountain codes that allows us to discard those parts of the channel in deep fading. Fountain codes can reconstruct the original source file by only collecting enough fountainencoded packets [6]. It does not matter which packet is received but we only need to receive enough packets. In other words, fountain-encoded packets are independent with respect to each other [6] [7]. Because fountain codes are designed for erasure channels, error correcting codes are required to transfer the noisy wireless channel into an erasure channel. If a fountain-encoded packet is transmitted over a single sub-carrier, we can reduce the dynamic range of channel by choosing between two approaches. Option I is to exchange the code rate of error correcting codes with the number of sub-carriers in deep fading; Option II is to sacrifice the subcarriers in deep fading by using a higher order modulation. In both cases, the receiver is allowed to discard fountain-encoded 


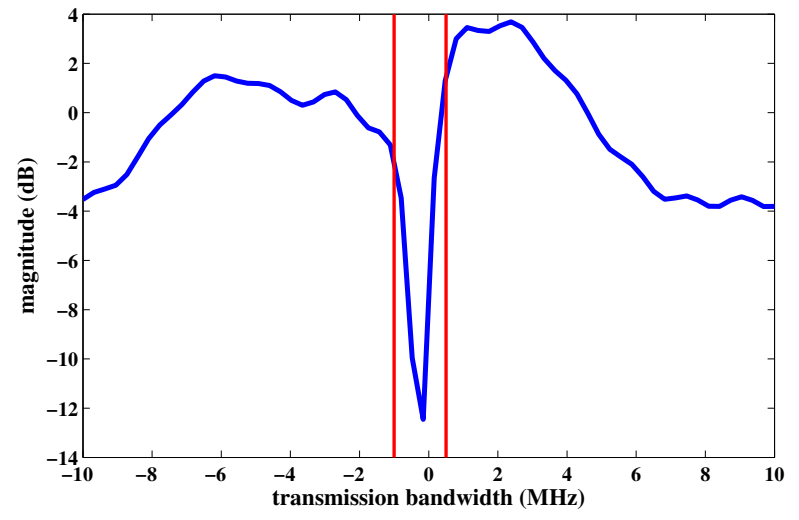

Fig. 1. Example of the baseband transfer function of a frequency selective Channel model A [5].

packets over the sub-carriers in deep fading. Correspondingly, the power consumption in ADCs decreases.

Opportunistic error correction is a novel cross coding scheme, as it combines the joint coding scheme and the separate coding scheme. Here, we call opportunistic error correction based on Option I as OEC-I and the one with Option II as OEC-II. In [8], we have investigated the performance of OEC-I. With the same throughput, simulation results showed that OEC-I saves more than $70 \%$ of energy in ADCs comparing to the IEEE 802.11a system [8]. However, the performance of OEC-II is still not clear. With convolutional codes, simulation results show that using 16-QAM instead of QPSK gives us a Signal-to-Noise Ratio (SNR) loss of around $6 \mathrm{~dB}$ (i.e. an increase in the ADC's resolution). That also gives us more discarded sub-carriers (i.e. a decrease in the ADC's resolution). Therefore, we investigate whether the tradeoff based on Option II is profitable in this paper.

The outline of this paper is as follows. We propose two techniques which together form OEC-II to reduce the power consumption: fountain codes and resolution adaptive ADCs. First, fountain codes are discussed which is followed by resolution adaptive ADCs. A practical example is given in this paper by considering the IEEE 802.11a system [9]. In section IV, the system model is depicted. Afterwards, simulation results are discussed, which compare OEC-II with the conventional 802.11a system. The paper ends with a discussion of conclusions and indication of future work.

\section{Fountain CODES}

OEC-II is based on fountain codes. Any fountain code (e.g. Luby Transform (LT) codes [10], Raptor Codes [11], etc) can be applied. In this paper, we choose LT codes to prove the concept of OEC-II.

Consider a block of size $K$ packets $s_{1}, s_{2}, \cdots, s_{K}$ to be encoded by a fountain code. A packet has $m$ bits and considered as a unit. A fountain-encoded packet is generated by selecting a set of source packets randomly and computing the bitwise sum (XOR) of these source packets [6]. Fountain codes can supply an unlimited stream of encoded packets based on $s_{1}, s_{2}, \cdots, s_{K}$. In practical systems, only a fixed number of packets $N_{t}$ is generated.

At the receiver, enough packets have to be received to reconstruct the original file. The required number of received packets $N\left(N \leq N_{t}\right)$ is slightly larger than $K$ and is defined by [6]:

$$
N=(1+\varepsilon) K
$$

where $\varepsilon$ is the percentage of extra packets and is called the overhead.

The mathematical principle behind the fountain decoding is to solve $K$ unknown parameters from $N$ linear equations. In principle, it can be solved by Gaussian elimination which has a high complexity. Therefore, the message-passing algorithm [12] is usually chosen to decode fountain codes. The messagepassing algorithm has a linear computation cost [6], but it requires a large $\varepsilon$ for small block size. For example, the practical overhead of LT codes is $14 \%$ when $K=2000$, which limits its application in the practical system [8]. By combining the message-passing algorithm with Gaussian elimination, the overhead of LT codes is reduced to $3 \%$ when $K \geq 500$ [8].

Fountain codes are designed for erasure channels. However, wireless channels are noisy channels. In practical systems, other error correction algorithms are used to convert the noisy channels into erasure channels, often Low-Density ParityCheck (LDPC) codes [12]. In this paper, LDPC codes are used together with a Cyclic Redundancy Check (CRC) to make the wireless channel behave like an erasure channel.

Our OEC-II encoding scheme has the following order. First, a fountain-encoded packet is created. Then, the CRC is added. Finally, the packet is encoded by the LDPC code.

At the receiver, each fountain-encoded packet is first LDPC decoded if its energy is above or equal to a threshold (i.e. corresponding to BER $\left.\leq 10^{-5}\right)^{1}$. The received packet is discarded if its energy is below the threshold. If the LDPC decoding fails, the received packet is discarded as well. If the LDPC decoding succeeds, the CRC is used to identify any errors undetected by the LDPC codes. If the CRC decoder detects an error, the receiver assumes that the whole packet has been lost. Once the receiver has obtained $N$ surviving fountain-encoded packets, it starts to recover the source data.

\section{RESOlution AdAPTIVE ADCs}

In the OFDM system, signal detection is performed in the frequency domain after equalization. It is not beforehand known how many ADC bits are required for proper detection, as quantization happens in the time domain. In this section, we derive a relation between the quantization noise in the time domain and the frequency domain for the OFDM system. After that, we present a scheme to design an optimum low-resolution ADC for the OFDM system in a certain channel condition.

\footnotetext{
${ }^{1}$ As the fountain-encoded packet in this paper has a short length (i.e. 312 bits), the LDPC decoding succeeds at a probability of more than $99.7 \%$ when $\mathrm{BER} \leq 10^{-5}$.
} 


\section{A. Minimum Number of Quantization Levels}

Because the quantization noise depends on the signal, we first analyze the statistical characteristics of the ADC input $r_{n}$. The channel is supposed to be noiseless, so the output at the $n_{t h}$ moment $r_{n}$ is defined as:

$$
r_{n}=\sum_{l=0}^{L-1} h_{l} x_{n-l}
$$

where $L$ is the number of channel taps, $h_{l}$ the channel tap and $x$ the transmitted signal. We assume that the quantization noise is dominant, so other noise (e.g. thermal noise) is ignored in this paper. From [13], we know that $x_{n}$ can be modeled as a complex Gaussian-distributed random variable with zero-mean and a variance of 1 .

According to the Central Limit Theorem, the probability density function of $r_{n}$ can be described as:

$$
f\left(r_{n}\right) \approx \frac{1}{\pi} e^{-\frac{\left|r_{n}\right|^{2}}{\sum_{l}\left|h_{l}\right|^{2}}}
$$

In other words, $r_{n} \sim \mathcal{C N}\left(0, \sum_{l}\left|h_{l}\right|^{2}\right)$.

The ADC output $y_{n}$ is expressed by:

$$
y_{n}=\mathcal{Q}\left(r_{n}\right)=\sum_{l} h_{l} x_{n-l}+n_{n}
$$

where $n_{n}$ is the quantization noise in the time domain. From [13], we know that $n_{n}$ is uniformly distributed with zero mean and a variance of $\frac{\Delta^{2}}{6}$, where $\Delta$ is the uniform quantization step.

After the OFDM demodulation, we obtain $Y_{k}$ as:

$$
\begin{aligned}
Y_{k} & =\frac{1}{\sqrt{\mathrm{N}}} \sum_{n} y_{n} e^{-j \frac{2 \pi}{\mathrm{N}} n k} \\
& =H_{k} X_{k}+N_{k}
\end{aligned}
$$

where $N_{k}$ is the quantization noise in the frequency domain and $H_{k}$ is the transfer function of the $k$-th sub-carrier defined by:

$$
H_{k}=\sum_{l} h_{l} e^{-j \frac{2 \pi}{\mathrm{N}} l k}
$$

In [13], the authors have shown that $N_{k}$ is a Gaussian distributed random variable with zero mean and a variance of $\frac{\Delta^{2}}{6}$. Thus, for each sub-carrier, the variance of the quantization noise is the same, but the Signal-to-(quantization)-Noise Ratio (SNR) is different due to different $H_{k}$ :

$$
\mathrm{SNR}_{k}=\frac{\left|H_{k}\right|^{2}}{\frac{\Delta^{2}}{6}}
$$

Error correcting codes are applied to mitigate the effects of quantization and each code has a certain SNR threshold to achieve a BER at a certain order (e.g. $10^{-5}$ ) or lower. So, the quantization step $\Delta$ can be determined once the error correcting code is chosen, the modulation type is decided and the channel is estimated.

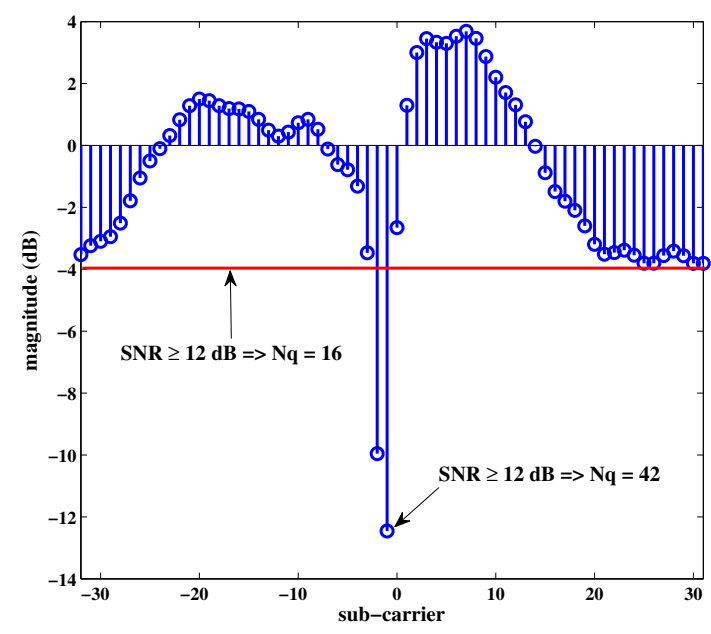

Fig. 2. The difference in the number of ADC levels $N_{q}$ between the transmission Scheme I and the transmission Scheme II.

If some clipping is allowed, the number of quantization levels $N_{q}$ is given by [13]:

$$
N_{q}=2\left\lceil\frac{\mathrm{C}}{\Delta}\right\rceil
$$

where $\mathrm{C}$ is equal to $3 \sigma_{r_{n}}$. For a given channel, $N_{q}$ only depends on $\Delta$. In such a case, $\Delta$ depends not only on the applied error correction codes and the chosen modulation type in the system, but also on how the encoded bits are transmitted.

Assume that fountain-encoded packets are transmitted over a wireless channel as shown in Fig. 1 and that a packet is received correctly when the $\mathrm{SNR} \geq 12 \mathrm{~dB}$. There are two schemes to transmit a fountain-encoded packet:

- Scheme I is to transmit a packet over all sub-carriers like current WLAN systems. In this case, the coding is done jointly. As we know, all sub-carriers have different energy. To make sure that the fountain-encoded packet can be correctly received, we should adapt the ADC's resolution to the worst case (i.e. the SNR of the worst sub-carrier should be at least equal to $12 \mathrm{~dB}$ ). Therefore, the required number of quantization levels $N_{q}$ for Scheme I is 42 for the example in Fig. 2.

- Scheme II transmits a packet over a single sub-carrier. Since each fountain-encoded packet is independent, it does not matter if we discard some packets which are transmitted over the sub-carriers in deep fading. From Fig. 2, we can see that by discarding 2 sub-carriers, $N_{q}$ can be reduced to 16 in comparison to Scheme I. The power consumption in a CMOS-based ADC scales linearly with $N_{q}$ [14]. In such case, discarding 2 subcarriers in this example saves us around $62 \%$ power in ADCs comparing to Scheme I. 


\section{B. Energy Consumption}

To receive a file, the energy consumption of the ADC is proportional to the number of quantization levels $N_{q}$ for each MAC frame and the total number of MAC frames $M_{c}$ used to transmit this file. In the WLAN system, the channel for each MAC frame is considered to be constant. $M_{c}$ depends on the size of file, the code rate of error correcting codes and the modulation type. It does not have to be an integral number. Thus, the energy consumption in ADCs to receive a certain amount of information is defined as:

$$
\mathrm{E}=\sum_{i=0}^{M_{c}-1} \alpha_{i} N_{q i} \mathcal{M}
$$

where $\alpha_{i}$ is the percentage of the $i$-th channel realization where useful information is transmitted, $N_{q i}$ is the number of quantization levels used in the $i$-th channel realization, and $\mathcal{M}$ is the number of samples per MAC frame.

\section{System Model}

OEC-II is based on fountain codes and resolution adaptive ADCs which have been explained in the above sections. OECII can be applied in the OFDM system. In this paper, the IEEE 802.11a system is taken as an example of OFDM systems.

The Forward Error Correction (FEC) layer in the current 802.11a system is based on Rate Compatible Punctured Convolutional (RCPC) codes. These codes only have good performance for random bit errors. Interleaving is employed to reduce burst errors. Each encoded packet is transmitted over all the sub-carriers, as the sub-carriers with high-energy can compensate for those with low-energy. Although this solution works well in practical systems, it is not energy efficient due to the following:

- The resolution of the applied ADCs is fixed for a 802.11a system.

- Even with a resolution adaptive ADC, the lowest resolution of ADCs required by the current FEC layer is dependent on the dynamic range of the channel.

- The receiver can not predict whether the received packet is likely to be decodable. That leads to a waste of processing power.

The above problems can be solved by OEC-II, as depicted in Fig. 3. The key idea is to increase the order of the modulation to compensate for the discard of the sub-carriers in deep fading. In such case, we can generate additional packets by the fountain encoder. First, source packets are encoded by a LT code, which is designed by using parameters $c=0.03$ and $\sigma=0.3$ [6]. Then, a 12-bit CRC checksum is added to each fountain-encoded packet before the LDPC decoding is applied. In this paper, we choose the $(324,648)$ LDPC code defined in the IEEE 802.11n standard [15] to encode each fountain-encoded packet. On each sub-carrier, a fountainencoded packet is transmitted. Thus, multiple packets are transmitted simultaneously, using frequency division multiplexing.
At the receiver side, we assume that the synchronization is perfect. With perfect channel estimation, the resolution of ADC is adapted to the minimum for the current channel condition according to Equation 7 and 8. However, the channel may not be perfectly estimated in practice. In this paper, we employ the zero-forcing algorithm to estimate the channel. A set of training symbols defined in [9] is used to estimate the channel. To reduce the channel estimation error, we set the ADCs in high-resolution mode to quantize the training symbols.

The $k_{t h}$ sub-carrier can be estimated by:

$$
\begin{aligned}
\hat{H}_{k} & =\frac{Y_{t}}{X_{t}} \\
& =H_{k}+\frac{N_{h}}{X_{t}}
\end{aligned}
$$

where $Y_{t}$ is the received training symbol, $X_{t}$ is the transmitted training symbol and $N_{h}$ is the quantization noise from highresolution ADCs. So, we can rewrite the output signal in the frequency domain after quantization defined in Equation 5 as:

$$
\begin{aligned}
Y_{k} & =H_{k} X_{k}+N_{a} \\
& =\hat{H}_{k} X_{k}-\frac{N_{h}}{X_{t}} X_{k}+N_{a} \\
& =\hat{H}_{k} X_{k}+N^{\prime}
\end{aligned}
$$

where $N_{a}$ is the quantization noise from resolution-adaptive ADCs. The variance $\sigma_{N^{\prime}}^{2}$ of $N^{\prime}$ is equal to $\sigma_{N_{h}}^{2}+\sigma_{N_{a}}^{2}$. Therefore, with the channel estimation error, the SNR for each sub-carrier defined in Equation 7 can be updated as:

$$
\begin{aligned}
\mathrm{SNR}_{k} & =\frac{\left|\hat{H}_{k}\right|^{2}}{\sigma_{N^{\prime}}^{2}} \\
& =\frac{\left|\hat{H}_{k}\right|^{2}}{\sigma_{N_{h}}^{2}+\sigma_{N_{a}}^{2}} \\
& =\frac{\left|\hat{H}_{k}\right|^{2}}{\sigma_{N_{h}}^{2}+\frac{\Delta^{2}}{6}}
\end{aligned}
$$

The channel estimation error will affect the SNR threshold for the correct LDPC decoding, as shown in Fig. 4. From this figure, we can see that the BER degradation can be neglected (within $0.1 \mathrm{~dB}$ for $\mathrm{BER} \leq 10^{-5}$ ).

\section{Performance Analysis}

In this section, we analyze the performance of OEC-II by comparing the following scenarios. Scenario I is a RCPC with interleaving from the IEEE 802.11a standard with $R_{\mathrm{I}}=0.5$ and QPSK as the modulation scheme. Fixed-resolution ADCs are used in Scenario I. As the standard allows $10 \%$ packet loss, the resolution of ADCs in Scenario I is designed for 90\% channel realizations. In Scenario II, we replace the fixedresolution ADCs in Scenario I by resolution adaptive ADCs. Scenario III is OEC-II based on fountain codes and resolution adaptive ADCs. Scenario III employs the same modulation scheme as Scenario I. In Scenario III, the code rate of LT code $R_{\mathrm{LT}}$ is around 0.97 and the code rate of LDPC plus CRC $R_{\mathrm{LDPC}-\mathrm{CRC}}$ is around 0.48 , so its total code rate $R_{\mathrm{III}}$ is 


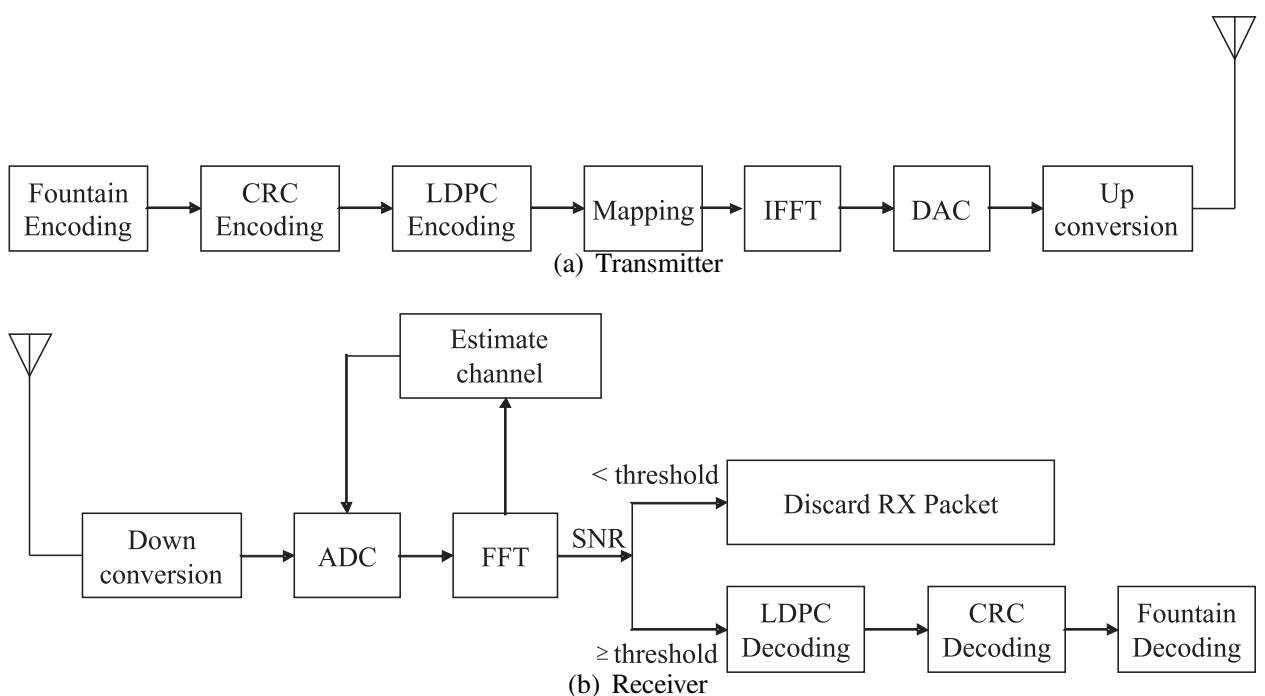

Fig. 3. The proposed IEEE 802.11a system with OEC-II: transmitter (top) and receiver (bottom).

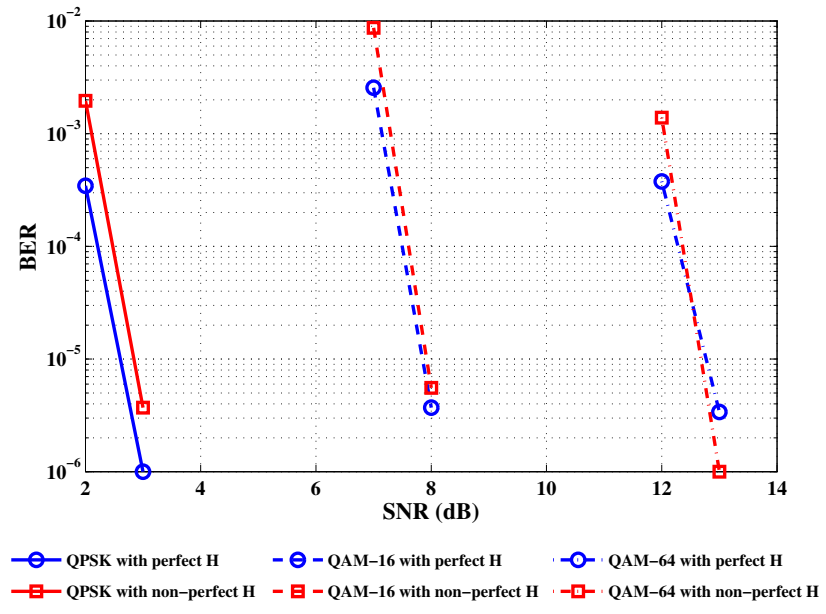

Fig. 4. Performance of the $(324,648)$ LDPC code with perfect channel knowledge and with non-perfect channel knowledge.

around 0.47. To have the same effective throughput, Scenario III is allowed to discard around $4 \%$ (i.e. $0.5 \times 0.9 / 0.47$ ) of data sub-carriers. Scenario IV is OEC-II with 16-QAM. In this case, around $52 \%$ (i.e. $(2 \times 0.5 \times 0.9) /(0.47 \times 4))$ of data sub-carriers can be discarded. Scenario $\mathbf{V}$ is OEC-II with 64-QAM which allows us to discard around $68 \%$ (i.e. $(2 \times$ $0.5 \times 0.9) /(0.47 \times 6))$ of data sub-carriers. In addition, 48 sub-carriers are used to transmit data as defined in [9].

Fig. 5 shows the average energy consumption in ADCs to receive a burst of data for two situations: with perfect channel knowledge and with non-perfect channel knowledge. Simulation results are based on the transmission of 1000 data bursts. Each burst consists of 168168 source bits (i.e. 539 packets with a length of 312 bits per packet) which are transmitted over 12 different channel realizations. For Scenario I and II, we use the corresponding BER value (i.e.

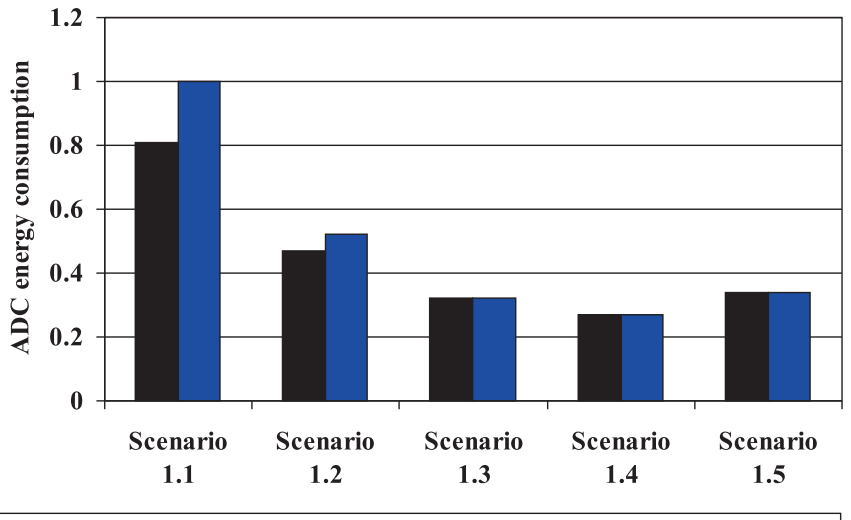

with perfect channel knowledge $\square$ with non-perfect channel knowledge

Fig. 5. Comparison in the ADC energy consumption between Scenario I, II, III, IV and V. They are compared in two situations: with perfect channel knowledge and with non-perfect channel knowledge. The energy consumption of Scenario I with non-perfect channel knowledge is normalized to 1 .

$\mathrm{BER} \approx 2.3 \times 10^{-4}$ as one packet is 54 Bytes) for the $10 \%$ packet loss. In such case, the sub-carrier with the lowest energy should have a SNR of $3.5 \mathrm{~dB}$. In the case of Scenario III, IV and $\mathrm{V}$, we assume that the received packets can be decoded successfully if the SNR is equal to or higher than the threshold (i.e. BER $\leq 10^{-5}$ ). Hence, all the wanted sub-carriers should be at least higher than the SNR threshold. From Fig. 4, we can see the SNR threshold for the situation with perfect channel knowledge is the same as the case with non-perfect channel knowledge (i.e. $3 \mathrm{~dB}$ for QPSK, $8 \mathrm{~dB}$ for 16-QAM and $13 \mathrm{~dB}$ for 64-QAM).

In Fig. 5, we normalize the energy consumption of Scenario I with non-perfect channel knowledge to $100 \%$. As we can see from this figure, the channel estimation error costs more ADC's energy in Scenario I and II, but that does not happen in the case of OEC-II (i.e. Scenario III, IV and V). Because 
the zero-forcing algorithm assumes no noise in the received signal, it performs better when the SNR is higher. Therefore, high-energy sub-carriers can be more accurately estimated than those in deep fading. In Scenario III, IV and V (i.e. OEC-II), we only need to take care of high-energy sub-carriers but we have to take care all the sub-carriers in the case of Scenario I and II. That explains OEC-II is less sensitive to the channel estimation error than the FEC layer in the 802.11a system.

In both situations, Scenario I costs the most ADC energy. Due to the resolution adaptive ADCs, Scenario II consumes less energy in ADCs than Scenario I. Resolution adaptive ADCs save around $42 \%$ energy in ADCs with perfect channel knowledge and around $48 \%$ power in the case of non-perfect channel knowledge. Fountain codes give a further power reduction in ADCs. With respect to the FEC layer from the IEEE 802.11a standard (i.e. Scenario II), OEC-II costs less energy in ADCs no matter which modulation type we use. In both situations, OEC-II with 16-QAM (i.e. Scenario IV) requires the least energy, which is followed by OEC-II with QPSK (i.e. Scenario III) then by OEC-II with 64-QAM (i.e. Scenario V). This shows the tradeoff between the ADC's energy consumption and the modulation order. Fountain codes with 16-QAM saves another $25 \%$ energy in ADCs in both situations. In total, OEC-II with 16-QAM (i.e. Scenario IV) reduces the power consumption in ADCs by around $67 \%$ with perfect channel knowledge and around $73 \%$ with non-perfect channel estimation comparing to Scenario I.

\section{CONCLUSIONS}

In this paper, we propose a novel cross-layer scheme to lower the power consumption of ADCs by combining a resolution adaptive ADC with OEC-II. Current OFDM systems employ fixed high-resolution ADCs which are designed for worst-case scenarios. Therefore, resolution adaptive ADCs can potentially save power in wireless receivers by tuning the ADC for each channel condition at run time. A further power reduction can be achieved by OEC-II based on fountain codes. The basic idea of OEC-II is to exchange the order of modulation with the discarded sub-carriers. By transmitting a fountain-encoded packet over a single sub-carrier, the receiver is allowed to discard packets over the sub-carriers in deep fading. Therefore, the ADC is allowed to take care of the subcarriers with high energy only. Correspondingly, the power consumption in ADCs decreases.

In this paper, we have investigated the performance of OEC-II in two situations: with perfect channel knowledge and with non-perfect channel knowledge. With respect to the conventional IEEE 802.11a system, resolution adaptive ADCs save around $42 \%$ energy in ADCs with perfect channel knowledge and around $48 \%$ energy in the case of non-perfect channel knowledge. In both situations, OEC-II with 16-QAM requires the least energy in $\mathrm{ADCs}$, which is followed by OEC-II with QPSK then by OEC-II with 64-QAM. Fountain codes with 16-QAM saves another $25 \%$ energy in ADCs in both situations. In total, OEC-II with 16-QAM reduces the energy consumption in ADCs by around $67 \%$ with perfect channel knowledge and around $73 \%$ with non-perfect channel estimation in comparison with the traditional 802.11a system.

The performance of OEC-I (i.e. to exchange the code rate of error correcting codes with the discarded sub-carriers) has been studied in [8] and evaluated practically in [16]. OECI saves more than $70 \%$ energy in the ADC comparing to the conventional 802.11a system. Therefore, further research focuses on the optimization of OEC by combining OEC-I and OEC-II together.

\section{ACKNOWLEDGEMENTS}

The authors acknowledge the Dutch Ministry of Economic Affairs under the IOP Generic Communication - Senter Novem Program for the financial support.

\section{REFERENCES}

[1] D. Tse and P. Viswanath, Fundamentals of Wireless Communication. New York, NY, USA: Cambridge University Press, 2005.

[2] J. Thomson and etc, "An Integrated 802.11 a Baseband and MAC Processor," in IEEE International Solid-State Circuits Conference (ISSCC), vol. 2, 2002.

[3] B. Murmann and B. Boser, Digitally Assisted Pipeline ADCs: Theory and Implementation. Kluwer Academic Pub, 2004.

[4] G. Moore et al., "Progress in Digital Integrated Electronics," SPIE MILESTONE SERIES MS, vol. 178, pp. 179-181, 2004.

[5] European Telecom Standards Institue, "Channel Models for HIPERLAN/2 in Different Indoor Scenarios," 1998.

[6] D.J.C. MacKay, "Fountain Codes," IEE Communications, vol. 152, no. 6, pp. 1062-1068, 2005.

[7] M. Mitzenmacher, "Digital Fountains: A Survey and Look Forward," in IEEE Information Theory Workshop, 2004, pp. 271-276.

[8] X. Shao and R. Schiphorst and C.H. Slump, "An Opportunistic Error Correction Layer for OFDM Systems," EURASIP Journal on Wireless Communications and Networking, 2009.

[9] IEEE, "Wireless LAN Medium Access Control (MAC) and Physical Layer (PHY) Specifications, High-Speed Pysical Layer in the $5 \mathrm{GHz}$ Band (IEEE 802.11a Standard, Part 11)," 1999.

[10] M. Luby, "LT Codes," Proceedings of the 43rd Annual IEEE Symposium on Foundations of Computer Science, pp. 271-282, 2002.

[11] A. Shokrollahi, "Raptor Codes," IEEE Transaction on Information Theory, vol. 52, 2006

[12] D.J.C. MacKay, Information Theory, Inference, and Learning Algorithms. Cambridge University Press, 2003.

[13] X. Shao and C.H. Slump, "Quantization Effects in OFDM Systems," in Proceedings of 29th Symposisum on Information Theory in the Benelux, Leuven, May 2008.

[14] S. Nahata, K. Choi, and S. Yoo, "A High-Speed Power and Resolution Adaptive Flash Analog-to-Digital Converter," in IEEE International SOC Conference, 12-15 Sept. 2004, pp. 33-36.

[15] IEEE, "Draft Standards for Wireless LAN Medium Access Control (MAC) and Physical Layer (PHY) Specifications, Enhancements for Higher Throughput (IEEE 802.11n Standard, Part 11)," Jan, 2007.

[16] X. Shao and C.H. Slump, "Practical Evaluation of Opportunistic Error Correction," in IEEE Global Communications Conference (GLOBECOM), December 2009, pp. 1-6. 